\title{
Effects of Dialysate Flow Configurations in Continuous Renal Replacement Therapy on Solute Removal: Computational Modeling
}

\author{
Jeong Chul Kim ${ }^{a-c}$ Dinna Cruz ${ }^{a, b}$ Francesco Garzotto ${ }^{a, b}$ Manish Kaushik ${ }^{a, b}$ \\ Catarina Teixeria $^{a}$ b Marie Baldwing lan Baldwing $^{9}$ Federico Nalesso $^{a, b}$ \\ Ji Hyun Kim ${ }^{d}$ Eungtaek Kang ${ }^{e}$ Hee Chan Kim ${ }^{c, f}$ Claudio Ronco ${ }^{a, b}$
}

a Department of Nephrology, Dialysis and Transplantation, San Bortolo Hospital, and ${ }^{\mathrm{b}}$ International Renal Research Institute Vicenza, Vicenza, Italy; ' Institute of Medical and Biological Engineering, Medical Research Center, Seoul National University, ${ }^{\mathrm{d}}$ Biomedical Research Institute, Center for Biomaterials, Korea Institute of Science and Technology, ${ }^{e}$ Department of Internal Medicine, Chung-Ang University Hospital, and ${ }^{\mathrm{f}}$ Department of Biomedical Engineering, College of Medicine, Seoul National University, Seoul, Korea; ${ }^{9}$ Department of Critical Care, Northern Hospital, Melbourne, Vic., Australia

\section{Key Words}

Acute kidney injury · Computational fluid dynamics • Continuous renal replacement therapy · Convection • Dialysis solutions $\cdot$ Diffusion $\cdot$ Solute clearance $\cdot$ Ultrafiltration

\begin{abstract}
Background/Aims: Continuous renal replacement therapy (CRRT) is commonly used for critically ill patients with acute kidney injury. During treatment, a slow dialysate flow rate can be applied to enhance diffusive solute removal. However, due to the lack of the rationale of the dialysate flow configuration (countercurrent or concurrent to blood flow), in clinical practice, the connection settings of a hemodiafilter are done depending on nurse preference or at random. Methods: In this study, we investigated the effects of flow configurations in a hemodiafilter during continuous venovenous hemodialysis on solute removal and fluid transport using computational fluid dynamic modeling. We solved the momentum equation coupling solute transport to predict quantitative diffusion and convection phenomena in a simplified hemodiafilter model. Results: Computational modeling results showed su-
\end{abstract}

\section{KARGER}

Fax +4161306 1234

E-Mail karger@karger.com

www.karger.com
(C) 2013 S. Karger AG, Basel

0253-5068/13/0353-0106\$38.00/0

Accessible online at:

www.karger.com/bpu perior solute removal (clearance of urea: $67.8 \mathrm{vs.} 45.1 \mathrm{ml} / \mathrm{min}$ ) and convection (filtration volume: 29.0 vs. $25.7 \mathrm{ml} / \mathrm{min}$ ) performances for the countercurrent flow configuration. Countercurrent flow configuration enhances convection and diffusion compared to concurrent flow configuration by increasing filtration volume and equilibrium concentration in the proximal part of a hemodiafilter and backfiltration of pure dialysate in the distal part. In clinical practice, the countercurrent dialysate flow configuration of a hemodiafilter could increase solute removal in CRRT. Nevertheless, while this configuration may become mandatory for high-efficiency treatments, the impact of differences in solute removal observed in slow continuous therapies may be less important. Under these circumstances, if continuous therapies are prescribed, some of the advantages of the concurrent configuration in terms of simpler circuit layout and simpler machine design may overcome the advantages in terms of solute clearance. Conclusion: Different dialysate flow configurations influence solute clearance and change major solute removal mechanisms in the proximal and distal parts of a hemodiafilter. Advantages of each configuration should be balanced against the overall performance of the treatment and its simplicity in terms of treatment delivery and circuit handling procedures.

Copyright $\odot 2013$ S. Karger AG, Basel

Claudio Ronco

Ospedale San Bortolo

Viale Rodolfi 37

IT-36100 Vicenza (Italy)

E-Mailcronco@goldnet.it 


\section{Introduction}

Continuous renal replacement therapy (CRRT) is commonly used in critically ill patients with acute kidney injury. In CRRT, blood flows into hollow fibers and dialysate into the space surrounding the fibers of a hemodiafilter. This provides a suitable environment for the diffusive and convective removal of wastes from a patient's blood as the fluid flows along the length of the fiber membrane (outside) to then be removed as waste fluid (effluent) at the opposing end. The connection of the dialysate fluid to the filter has traditionally been applied such that the fluid flows in a direction opposite to the blood flow (countercurrent direction) in order to provide maximum small solute clearance by diffusion. However, while this is based on evidence during conventional hemodialysis treatment, and thought to be best at very high dialysate flow rates $\left(\mathrm{Q}_{\mathrm{D}}=500 \mathrm{ml} / \mathrm{min}\right)$, at low dialysate flow rates (such as those used in CRRT) with apparently complete saturation of the dialysate with an effluent to plasma ratio of around 1.0 [1], the evidence of superiority of one configuration over the other is missing. There are no data to indicate that the countercurrent dialysate flow configuration should be preferred in CRRT given the low dialysate flow rates normally used $\left(\mathrm{Q}_{\mathrm{D}}<50 \mathrm{ml} / \mathrm{min}\right)$. This aspect may become a matter of debate in case of new hardware design and development since some advantages may be seen in the concurrent configuration (easier priming, easier handling of the circuit, and simpler layout of blood lines and extracorporeal circuit).

Some studies have addressed the effects of flow configurations in a hemodiafilter on solute removal in extracorporeal renal replacement therapy. A simple theoretical analysis of one-dimensional flow along parallel tubes showed that the total transfer of solute is always more efficient with a countercurrent than concurrent configuration [2]. However, based on this analytical solution, the difference in solute extraction using CRRT with different flow configurations was less than $5 \%$. An in vitro study with two flat polyethersulfone membranes with filtration cutoff levels of around 40 and $500 \mathrm{kDa}$, clearance rates of urea (molecular weight, MW, 60) and vitamin B12 (MW $1,355)$ were higher with the countercurrent flow configuration than with the concurrent one [3]. As MW increases, difference in clearance also increased. Another in vitro study with a low-flux hemodialyzer also revealed that changing the countercurrent flow to concurrent deteriorates diffusive clearance rates of bicarbonate (MW 61) and trisodium phosphate (MW 163.94) by 14 and $18 \%$, respectively [4]. However, these studies have been done for high dialysate flows or high dialysate/blood flow ratio. Because, in CRRT, dialysate flow is $1,000-2500 \mathrm{ml} / \mathrm{h}$ and the dialysate/blood ratio is less than 0.2 , different results may be expected with less difference in solute extraction with the two configurations. A clinical study of continuous arteriovenous hemodialysis showed a greater pressure drop in the blood compartment of the hemodiafilter and significantly higher clearance rates of urea and creatinine in the countercurrent dialysate flow configuration [5]. However, the rate of flow blood in continuous arteriovenous hemodialysis was not constant like that in continuous venovenous hemodialysis (CVVHD) and the ultrafiltration rate was not strictly controlled due to the lack of real-time ultrafiltration feedback control making it difficult to directly apply the results in clinical practice.

Current CRRT machines used in the intensive care unit are equipped with a continuous ultrafiltration control system and enable dialysate flow to be connected both in countercurrent and concurrent flow configurations. Extracorporeal settings of dialysate flow direction are done depending upon nurse preference or at random. However, flow configurations of blood and dialysate flow could influence pressure profiles in a hemodiafilter, resulting in different convection and solute diffusion profiles during CRRT. Currently, we have no data to support the rationale for a countercurrent dialysate configuration in terms of solute removal and fluid transport. In this study, we investigated the effects of flow configurations in a hemodiafilter on solute removal and fluid transport during CRRT using computational fluid dynamic analysis.

\section{Materials and Methods}

An equivalent annulus model was adapted [6] to simulate diffusion and convection during CVVHD. Axisymmetric computational meshes of blood and dialysate compartments were constructed including porous hollow fiber membrane. Specification and physical properties of the hemodiafilter are listed in table 1. The equivalent diameter of the dialysate compartment was calculated from the thickness of the hollow fiber membrane and fiber packing density. Governing equations were adopted from a previous simulation study on a hollow fiber membrane of newtonian fluids for laminar, incompressible, isothermal and fully developed flow in a single hollow fiber system using computational fluid dynamics software (FLUENT 6.2; Fluent, Inc., Lebanon, N.H., USA).

Conservation of mass:

$$
\frac{\partial \rho}{\partial t}+\frac{\partial}{\partial x_{i}}\left(\rho u_{i}\right)=0
$$




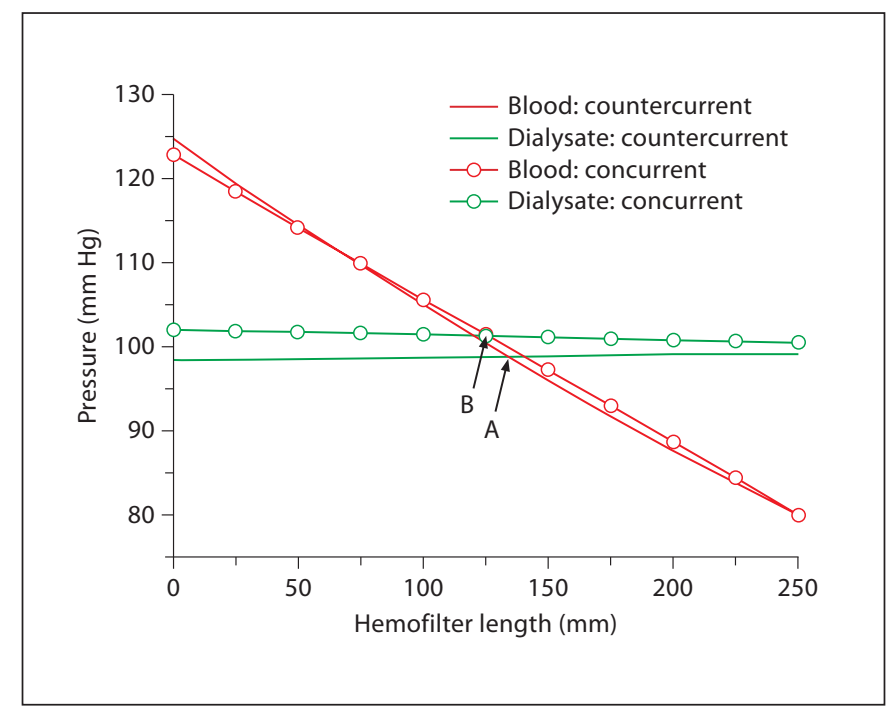

Fig. 1. Pressure profiles in blood and dialysate compartments of a hemodiafilter. A: pressure equilibrium point for the countercurrent flow configuration; B: pressure equilibrium point for the concurrent flow configuration.

Table 1. Specifications and physical properties of the membrane of a hemodiafilter used in computational modeling

\begin{tabular}{ll}
\hline Surface area & $1.3 \mathrm{~m}^{2}$ \\
Fiber inner diameter & $200 \mu \mathrm{m}$ \\
Fiber thickness & $40 \mu \mathrm{m}$ \\
Fiber length & $250 \mathrm{~mm}$ \\
Fiber packing density & 0.5 \\
Ultrafiltration coefficient & $60 \mathrm{ml} / \mathrm{h} / \mathrm{mm} \mathrm{Hg}$ \\
\hline
\end{tabular}

Table 2. Calculated hydrodynamic parameters during CVVHD

\begin{tabular}{llcl}
\hline Parameter & $\begin{array}{l}\text { Counter- } \\
\text { current }\end{array}$ & $\begin{array}{l}\text { Con- } \\
\text { current }\end{array}$ & Reference \\
\hline$\Delta \mathrm{P}_{\mathrm{B}}, \mathrm{mm} \mathrm{Hg}$ & 44.7 & 42.9 & - \\
$\Delta \mathrm{P}_{\mathrm{D}}, \mathrm{mm} \mathrm{Hg}$ & 1.0 & 1.5 & - \\
Transmembrane pressure, $\mathrm{mm} \mathrm{Hg}$ & 7.21 & -0.35 & $0-20^{1}$ \\
Total filtration rate, $\mathrm{ml} / \mathrm{min}$ & 29.0 & 25.7 & $30-40^{2}$ \\
\hline
\end{tabular}

\footnotetext{
${ }^{1}$ Clinical observation.

${ }^{2}$ Approximated from the ultrafiltration coefficient of membrane and backfiltration rate at $\mathrm{Q}_{\mathrm{B}}=300 \mathrm{ml} / \mathrm{min}$ and $\mathrm{Q}_{\mathrm{D}}=500$ $\mathrm{ml} / \mathrm{min}$.
}

Conservation of momentum:

$$
\frac{\partial}{\partial t}\left(\rho u_{i}\right)+\frac{\partial}{\partial x_{i}}\left(\rho u_{i} u_{j}\right)=-\frac{\partial p}{\partial x_{i}}+\frac{\partial \tau_{i j}}{\partial x_{i}}+\rho g_{i}
$$

Solute transport:

$$
J=-\rho D_{i} Y_{i}
$$

where $\rho$ is the density of the fluid, $u_{i}$ is the tensor notation of velocity components, $p$ is the static pressure, $\tau_{i j}$ is the stress tensor, $\rho g_{i}$ is the gravitational body force term, $J$ is the solute flux, $D_{i}$ is the diffusion coefficient of solute and $Y_{i}$ is the concentration of the solute.

Physical properties of blood and dialysate were assumed as follows: $\mu_{b}=3.5 \mathrm{cP}, \rho_{b}=1,060 \mathrm{~kg} / \mathrm{m}^{3}, \mu_{d}=0.84 \mathrm{cP}$ and $\rho_{b}=999$ $\mathrm{kg} / \mathrm{m}^{3}$. Oncotic pressure was set as a constant of $25 \mathrm{~mm} \mathrm{Hg}$. As boundary conditions for simulation, a blood flow rate of $200 \mathrm{ml} /$ $\mathrm{min}$ and a dialysate flow rate of $2,500 \mathrm{ml} / \mathrm{h}$ were applied to simulate the operating conditions of CVVHD. We assumed $P_{B o}$ of 80 $\mathrm{mm} \mathrm{Hg}$ based on clinical observation. $P_{D o}$ was adjusted to meet zero ultrafiltration condition, and viscosity change by hemoconcentration was not considered because the main purpose of our study was to evaluate the relative solute transport. Porous media were modeled based on Darcy's law, and the viscous resistance was calculated based on the ultrafiltration coefficient and membrane thickness.

We set the diffusion coefficient of urea (MW 60) at $1.0 \times 10^{-9}$ $\mathrm{m}^{2} / \mathrm{s}$ (adapted from previous experimental values measured in flowing blood) [7] and the diffusion coefficient of creatinine (MW 113) was set to $5.31 \times 10^{-10} \mathrm{~m}^{2} / \mathrm{s}$ based on the linear correlation between diffusion coefficient and MW for small molecules [8]. For $\beta_{2}$-microglobulin, we used the experimentally measured diffusion coefficient of cytochrome C (MW 13,370): $1.05 \times 10^{-10}$ $\mathrm{m}^{2} / \mathrm{s}$ [9]. Blood inlet concentrations of solutes for the computational model were $100 \mathrm{mg} / \mathrm{dl}$ for urea, $2.0 \mathrm{mg} / \mathrm{dl}$ for creatinine and $10 \mathrm{mg} / \mathrm{l}$ for $\beta_{2}$-microglobulin. Sieving coefficients of all solutes were assumed as 1.0.

To validate the computational model, we compared hydrodynamic parameters (transmembrane pressure and filtration volume) with previous in vitro [10] and clinical observations. Solute transport parameters (extraction ratio, effluent/plasma ratio and clearance) were compared with the results of a previous study under similar treatment conditions [11].

\section{Results}

Pressure profiles in blood and dialysate compartments during CVVHD are shown in figure 1. A higher pressure drop in the blood compartment was noted for the countercurrent than for the concurrent flow configuration (table 2). The difference in the pressure drop in the dialysate compartment between the different flow configurations was negligible. While a nonlinear pressure curve in the blood compartment was observed in the countercurrent flow configuration, a linear pressure profile was observed in the concurrent flow configuration. Therefore, trans- 

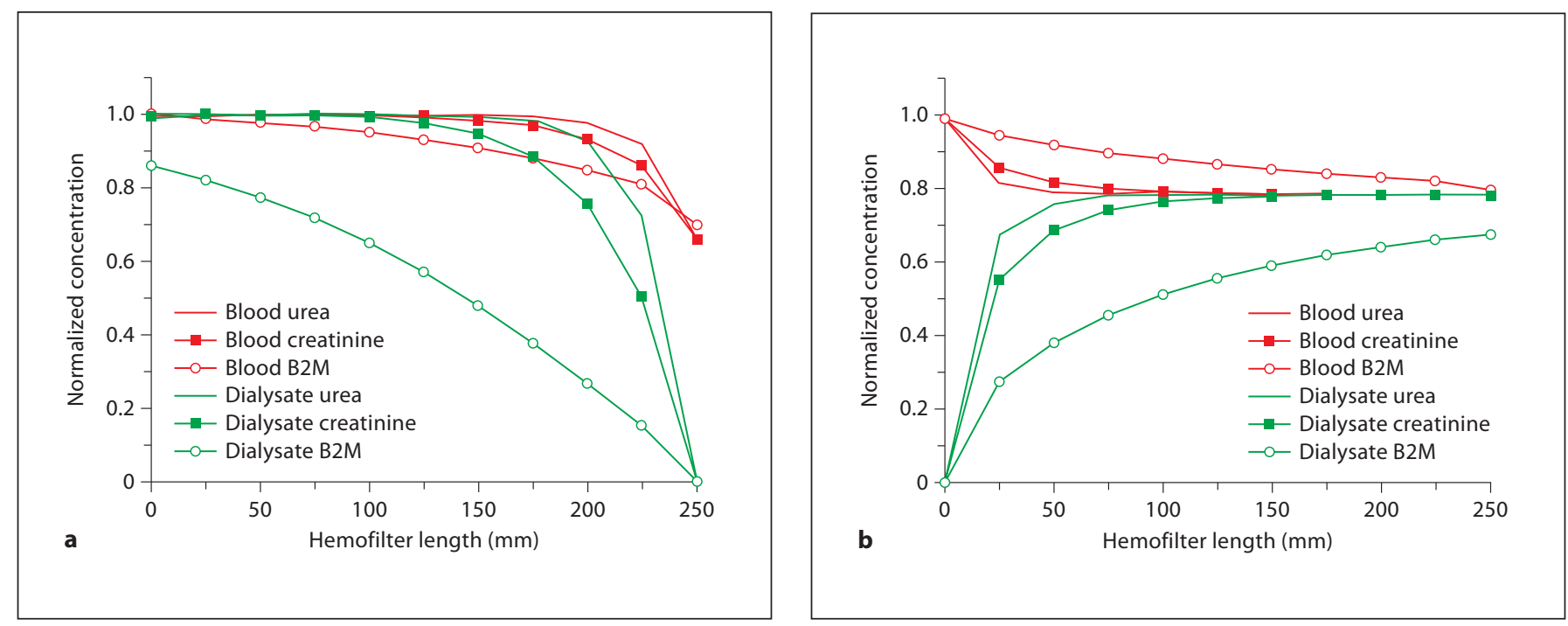

Fig. 2. Normalized concentration profiles of urea, creatinine and $\beta_{2}$-microglobulin (B2M) in blood and dialysate compartments of a hemodiafilter. a Countercurrent dialysate flow configuration. b Concurrent dialysate flow configuration.

Table 3. Calculated solute removal parameters during CVVHD

\begin{tabular}{|c|c|c|c|c|c|c|c|c|c|}
\hline $\begin{array}{l}\text { Diffusion } \\
\text { coefficient }\end{array}$ & \multicolumn{3}{|l|}{ Urea } & \multicolumn{3}{|c|}{ Creatinine } & \multicolumn{3}{|c|}{$\beta_{2}$-Microglobulin } \\
\hline Extraction ratio, \% & 33.9 & 22.6 & - & 33.8 & 22.4 & - & 30.1 & 17.9 & - \\
\hline Effluent/plasma ratio & 1.0 & 0.77 & - & 0.99 & 0.76 & - & 0.86 & 0.50 & - \\
\hline Clearance, $\mathrm{ml} / \mathrm{min}$ & 67.8 & 45.1 & $45.1(40.5,56.4)$ & 67.6 & 44.7 & $59.3(50.1,64.5)$ & 60.1 & 35.7 & $48.8(45.8,60.2)$ \\
\hline
\end{tabular}

${ }^{1}$ Clearance measured at $\mathrm{Q}_{\mathrm{B}}=200 \mathrm{ml} / \mathrm{min}$ and $\mathrm{Q}_{\mathrm{D}}=2,500 \mathrm{ml} / \mathrm{min}$ in the countercurrent dialysate flow configuration.

membrane pressure and total filtration rate (internal filtration + backfiltration) in the hemodiafilter were higher for the countercurrent than for the concurrent flow configuration, which were comparable with a dialysate flow rate of $33.3 \mathrm{ml} / \mathrm{min}$. The pressure equilibrium points were in the distal part of the hemodiafilter for countercurrent flow but at the half of the hemodiafilter for concurrent flow (see A and B in fig. 1).

Solute concentration profiles according to $\mathrm{MW}$ in blood and dialysate compartments are shown in figure 2 . In both concurrent and countercurrent flow configurations, solute concentrations of urea and creatinine reached the equilibrium in the distal part of the hemodiafilter while a disequilibrium remained with respect to the $\beta_{2^{-}}$ microglobulin concentrations. The normalized equilib- rium concentration of urea was 1.0 for concurrent flow and 0.76 for countercurrent flow. Table 3 shows the extraction ratio, effluent/plasma ratio and clearance for urea, creatinine and $\beta_{2}$-microglobulin. Results show that solute removal rate is always higher in countercurrent than concurrent flow for both small and large solutes by $63.1 \%$ (urea), 62.6\% (creatinine) and 52.7\% ( $\beta_{2}$-microglobulin). As the diffusion coefficient of solute decreased (i.e. MW increased), the difference in solute removal between countercurrent and concurrent flow configurations decreased. Comparing computational modeling results with results of previous clinical studies, solute clearance was overestimated by $33 \%$ (urea), 12\% (creatinine) and $18 \%\left(\beta_{2}\right.$-microglobulin). 


\section{Discussion}

In this study, we investigated the effects of dialysate flow configurations (counter- vs. concurrent) in a hemodiafilter during CRRT on solute removal and fluid transport using computational fluid dynamic modeling. We solved the momentum equation coupling solute transport to predict quantitative diffusion and convection phenomena in a simplified hemodiafilter model. During CVVHD, the computational model predicted convection and diffusion phenomena with the allowable errors compared with clinical observation and previous clinical study. Simulation results showed superior solute removal performances for countercurrent flow, which was attributed to the larger filtration volume and higher equilibrium concentrations between blood and dialysate compartments.

Although the computational model predicted convection transport in a hemodiafilter accurately, solute clearance was overestimated in the model (table 3). The differences in solute clearance between computational modeling and clinical results are, possibly, attributable to nonuniform flow distributions and blood-dialysis membrane interaction. While uniform blood and dialysate flow distributions are assumed, in clinical practice, those in hollow-fiber hemodialyzers are not uniform, which results in flow mismatch that impairs diffusion transport across the dialysis membrane [12]. Blood-membrane interaction is another factor that leads to reductions in diffusion and convection transport in the dialysis membrane [13]. Concentration polarization and protein layers which developed on the surface of the dialysis membrane as well as pore plugging by proteins increase effective transmembrane pressure to maintain the prescribed ultrafiltration rate and decrease the effective membrane surface area for solute and fluid transport, possibly explaining the discrepancy between the prescribed and the delivered dose in CRRT $[14,15]$. As the diffusion coefficient decreased (and MW increased), the difference in solute clearance between counter- and concurrent flow configurations decreased, which is contrary to previous in vitro studies reporting higher differences in the clearance of larger molecules $[3,4]$.

Another factor that may influence solute transport in extracorporeal renal replacement therapy is gravity. According to Hagen-Poiseuille's law, a pressure drop in pipe flow can be expressed as

$$
P_{\text {in }}-P_{\text {out }}=\frac{128 \mu L Q}{\pi d^{4}}+\rho g L
$$

The sign convention of gravity is positive when the flow direction is upward and negative for downward blood flow direction. Considering physical blood properties, the second term is about $18 \mathrm{~mm} \mathrm{Hg}$ and the difference between upward and downward flow direction is 2 times this value. The momentum effect in the dialysate compartment is negligible compared to that of the blood compartment due to lower viscosity of the dialysate, a low flow rate of dialysate in CRRT and its larger hydraulic perimeter. For the upward blood flow setting, the pressure in the blood compartment is higher than for the downward blood flow setting and, consequently, the mean pressure of the dialysate increases to meet the prescribed ultrafiltration rate in the hemodiafilter. However, this increase in pressure levels due to gravity does not significantly influence convection and diffusion phenomena in the hemodiafilter because driving forces are not represented by absolute levels but rather by differences in static pressure and concentration.

In spite of these considerations, we may argue that there are potential advantages of the concurrent dialysate flow configuration. It is important to remove air bubbles in both blood and dialysate compartments during hemodiafilter priming and treatment, and this may be easier if a concurrent configuration is selected. Kurusz et al. [16] suggested that air emboli activate the coagulation process. Air emboli represent a foreign surface to blood. Activation of both cellular and noncellular components occurs and may have consequences for the patient after the disappearance of the bubbles. Coagulation of hemodiafilter air bubbles in the dialysate compartment results in a decrease in the effective surface for diffusion and convection and also impairs dialysis adequacy. Microemboli developing during hemodialysis are not trapped by the drip chamber and might cause microischemic lesions in organs such as the lung and brain [17]. Microemboli can develop in the hemodialyzer [18] when unstable hydrodynamic processes are involved, such as backfiltration and incomplete priming. Concurrent dialysate flow can continuously remove air bubbles in the hemodiafilter when the flow directions are upwards, contrary to gravity. Additionally, when blood and dialysate flow in the same direction, more natural and stable hydrodynamic conditions are achieved and more reliable CRRT can be performed for a long time without frequent interruption and control of the effluent pump of the machine. Finally, the concurrent configuration may result in a simpler blood and dialysate line layout improving simplicity of the extracorporeal circuit and hemodiafilter priming and handling. In case of new hardware design and development, 
the concurrent configuration may result in a simpler machine layout and circuit design.

Considering convection and diffusion phenomena in a hemodiafilter together (fig. 1, 2), for the countercurrent flow configuration, in the proximal part of a hemodiafilter, internal filtration is the major solute removal mechanism with low diffusion due to saturated dialysate solute concentration while in the distal part of a hemodiafilter, fresh dialysate is backfiltered into the blood compartment with minor diffusion between blood and dialysate. In case of the concurrent flow configuration, on the contrary, in the proximal part of a hemodiafilter convection and diffusion occur simultaneously while in the distal part, minor diffusion and backfiltration of dialysate mixed with filtrate occurs. In conclusion, the countercurrent flow configuration in CRRT enhances convection and diffusion compared to the concurrent flow configuration by increasing fluid exchange volume and equilibrium concentration. In clinical practice, the countercurrent flow configuration setting could increase solute removal although potential advantages of a concurrent configuration layout should be considered. Future clinical studies should compare these two configurations in terms of solute removal in CRRT analyzing also other hemodynamic and hydrodynamic parameters. In the clinical setting, small differences in solute clearance should be balanced against advantages of the concurrent configuration in terms of handling and more effective delivery of therapy. It is possible that in spite of a given superiority of the countercurrent configuration in terms of kinetics of solute exchanges, the final clinical effect may result marginally different making the configuration easier to handle more suitable for routine treatment. These studies will represent important indications for manufacturers engaged in new hardware design, and for operators (physicians and nurses) engaged in the prescription and delivery of CRRT.

\section{Acknowledgment}

This study was supported by a Fondazione Cariverona grant (No. 2010.1087).

\section{Disclosure Statement}

The authors have no competing interests.

\section{References}

1 Claure-Del Granado R, Macedo E, Chertow GM, Soroko S, Himmelfarb J, Ikizler TA, Paganini EP, Mehta RL: Effluent volume in continuous renal replacement therapy overestimates the delivered dose of dialysis. Clin J Am Soc Nephrol 2011;6:467-475.

2 Keener J, Sneyd J: Renal physiology; in Keener J, Sneyd J (eds): Mathematical Physiology. Berlin, Springer, 1998, pp 421-450.

-3 Ould-Dris A, Paullier P, Griscom L, Legallais C, Leclerc E: Analysis of the mass transfers in an artificial kidney microchip. J Membr Sci 2010;352:116-125.

-4 Eloot S, De Vos JY, Hombrouckx R, Verdonck P: Diffusive clearance of small and middle-sized molecules in combined dialyzer flow configurations. Int J Artif Organs 2004;27:205-213.

5 Davenport A, Will EJ, Davison AM: Effect of the direction of dialysate flow on the efficiency of continuous arteriovenous haemodialysis. Blood Purif 1990;8:329-336.

-6 Liao Z, Klein E, Poh CK, Huang Z, Hardy PA, Morti S, Clark WR, Gao D: A modified equivalent annulus model for the hollow fiber hemodialyzer. Int J Artif Organs 2004; 27:110-117.

Flow Configurations in CRRT
7 Colton CK, Smith KA, Merrill ER, Friedman S: Diffusion of urea in flowing blood. AIChE J 1971;17:800-808.

8 Valencia DP, González FJ: Understanding the linear correlation between diffusion coefficient and molecular weight. A model to estimate diffusion coefficients in acetonitrile solutions. Electrochem Commun 2011; 13:129-132.

-9 Young ME, Carroad PA, Bell RL: Estimation of diffusion coefficients of proteins. Biotechnol Bioengin 1980;22:947-955.

$\checkmark 10$ Raff M, Welsch M, Göhl H, Hildwein H, Storr M, Wittner B: Advanced modeling of highflux hemodialysis. J Membr Sci 2003; 216:1-11.

11 Floris M, Marchionna N, Clementi A, Kim JC, Cruz DN, Nalesso F, Zanella M, Garzotto F, de Cal M, Virzi GM, Brendolan A, Ronco C: Evaluation of a new polysulfone hemofilter for continuous renal replacement therapy. Blood Purif 2011;32:133-138.

12 Ronco C, Brendolan A, Crepaldi C, Rodighiero M, Scabardi M: Blood and dialysate flow distributions in hollow-fiber hemodialyzers analyzed by computerized helical scanning technique. J Am Soc Nephrol 2002;13(suppl 1):S53-S61
13 Huang Z, Gao D, Letteri J, Clark WR: Blood-membrane interactions during dialysis. Semin Dial 2009;22:623-628.

14 Vesconi S, Cruz DN, Fumagalli R, KindgenMilles D, Monti G, Marinho A, Mariano F, Formica M, Marchesi M, Rene R, Livigni S, Ronco C: Delivered dose of renal replacement therapy and mortality in critically ill patients with acute kidney injury. Crit Care 2009;13:R57.

15 Venkataraman R, Kellum JA, Palevsky P: Dosing patterns for continuous renal replacement therapy at a large academic medical center in the United States. J Crit Care 2002;17:246-250.

16 Kurusz M, Butler B, Katz J, Conti VR: Air embolism during cardiopulmonary bypass. Perfusion 1995;10:361-391.

- 17 Forsberg U, Jonsson P, Stegmayr C, Stegmayr B: Microemboli, developed during haemodialysis, pass the lung barrier and may cause ischaemic lesions in organs such as the brain. Nephrol Dial Transplant 2010;25:2691-2695.

18 Stegmayr C, Jonsson P, Forsberg U, Stegmayr B: Hemodialysis dialyzers contribute to contamination of air microemboli that bypass the alarm system in the air trap. Int J Artif Organs 2008;31:317-322. 